\title{
Nanostructure and optical transparency of silica aerogels
}

\author{
A. EMMERLING, P. WANG, G. POPP, A. BECK and J. FRICKE
}

Physikalisches Institut der Universität, Am Hubland, 97074 Würzburg, Germany

\begin{abstract}
Silica aerogels are promising materials for transparent thermal insulation systems in solar architecture. The optical transparency of these highly porous substances is influenced by the reaction parameters upon preparation. The specific extinction due to bulk scattering was found to decrease both with increasing macroscopic density and increasing $\mathrm{pH}$-value of the sol-gel starting solution. Since the inhomogeneities of the aerogel network are much smaller than the light wavelengths, nearly isotropic light (Rayleigh) scattering is expected and observed. In order to check correlations between optical transparency and nanostructural build-up of the aerogels, small angle X-ray scattering measurements have been performed. Both, X-ray and light scattered intensity, have been reduced to an absolute scale. Within the measurement accuracy the light scattering intensity of the aerogel bulk is equal to the extrapolated X-ray scattering intensity towards scattering angle zero. As a consequence, the amount of isotropically scattered light and thus the optical extinction can directly be related to the correlation volume. It will be shown how the latter depends on the nanostructural features of the gel network, such as average particle size, interparticle arrangement, pore diameter and an ordering parameter, which accounts for concentration effects.
\end{abstract}

\section{Introduction}

Silica aerogels are sol-gel derived, supercritically dried materials with extraordinary properties due to their highly porous nanostructure [1]: Small sound velocities $(100 \mathrm{~m} / \mathrm{s}$ for an aerogel with macroscopic density of $100 \mathrm{~kg} / \mathrm{m}^{3}$ ), high accessible specific surfaces (up to $1000 \mathrm{~m}^{2} / \mathrm{g}$ ) and low thermal conductivities (below $0.02 \mathrm{Wm}^{-1} \mathrm{~K}^{-1}$ at room temperature in air). In addition, silica aerogels are transparent in the visible region which makes them promising materials for use as transparent insulation in solar architecture [2,3]. The use of monolithic aerogels in windows, however, requires a considerable improvement of the transparency. Since absorption of silica is negligible in the visible range, the extinction of light solely results from scattering. Recently, two different sources of light scattering in aerogels have been found [4]: Scattering due to $\mu \mathrm{m}$-sized imperfections of the external aerogel surface, which is the reason for the slightly fuzzy appearance of objects viewed through a piece of aerogel, and scattering from the aerogel bulk. The latter was shown to vary drastically upon silica content and catalyst concentration of the sol-gel starting solution. The question arises, whether the optical transparency can be correlated to the structural build-up of the gel network as derived from small angle $X$-ray scattering measurements.

\section{Theory}

The intensity measured in a scattering experiment is given by (see e.g. [5])

$$
I(q)=I_{0} T(q) V \frac{d \Sigma}{d \Omega}(q) \Delta \Omega w=f \frac{d \Sigma}{d \Omega}(q)
$$

$\mathrm{I}_{0}$ is the incident intensity, $\mathrm{T}$ the sample transmission, $\mathrm{V}$ the illuminated volume, $\Delta \mathbf{Q}$ and $\mathbf{w}$ are the solid angle and sensitivity of the detector, respectively, and $q=(4 \pi / \lambda) \sin \theta$ is the scattering vector, with $\lambda$ being the wavelength used and $2 \theta$ the scattering angle. The volume-specific scattering cross section

$$
\frac{\mathrm{d} \Sigma}{\mathrm{d} \Omega}(q)=\delta^{2} \int_{0}^{\infty} 4 \pi \mathrm{r}^{2} \gamma(r) \frac{\sin q \mathrm{r}}{\mathrm{qr}} \mathrm{dr}=\delta^{2} \mathrm{~V}_{\mathrm{c}}(\mathrm{q})
$$

depends on the mean square fluctuation of the scattering length density $\delta^{2}$ and the integral over the correlation function $\gamma(r)$, which contains the information of the geometric build-up of the sample. The polarisation factor can be omitted, if only the component of scattered light perpendicular to the scattering plane is considered, and neglected in case of small angle scattering. In the following, the integral term is abbreviated by $V_{c}(q)$, i.e. a $q$-dependent volume in which coherent scattering takes place. In case of $\mathrm{X}$-ray scattering $(\mathrm{X}), \delta^{2} \mathrm{X}$ is given by the product of the square of the classical electron radius $r_{e}$ times the mean square fluctuation in electron density $\eta^{2} \mathrm{X}$. For light scattering in the Rayleigh-Gans approximation [6], $\delta^{2}$ is equal to $\pi^{2} \eta_{L}^{2} / \lambda^{4}$ where $\eta_{L}^{2}$ denotes the mean square fluctuation of the dielectric constant. For a two-phase media system such as an aerogel, $\eta_{L}^{2}$ can be calculated from 


$$
\eta_{L}^{2}=\phi\left(\varepsilon_{\mathrm{S}}-\varepsilon\right)^{2}+(1-\phi)(\varepsilon-1)^{2},
$$

with $\varepsilon_{\mathrm{S}}$ being the dielectric constant and $\phi=\rho / \rho_{\mathrm{S}}$ the volume fraction of the solid phase; $\rho$ and $\rho_{\mathrm{S}}$ are the macroscopic and skeletal density of the aerogel, respectively. The average dielectric constant of the porous medium $\varepsilon$ can be obtained from the Clausius-Mosotti-relation using the values of nonporous vitreous silica $[6,7]$.

The optical extinction $E$ is related to the differential cross section and the measured light scattering intensity via

$$
E=\int_{4 \pi} \frac{d \Sigma_{L}}{d \Omega}(q) d \Omega=\frac{1}{f} \int_{4 \pi} I_{L}(q) d \Omega=\frac{1}{f} E^{*},
$$

with $f$ containing all (unknown) experimental parameters. By determination of the extinction $E$, for instance, from a measurement of the directional-directional transmission of the sample ( $T=e^{-E d}$, with sample thickness $d$ ) and calculation of the auxiliary extinction $E^{*}$ from the scattered intensity $I_{L}(q)$ measured in arbitrary units, it is possible to obtain the volume-specific specific differential scattering cross section of the sample:

$$
\frac{d \Sigma_{L}}{d \Omega}(q)=\frac{E}{E^{*}} I_{L}(q)
$$

If isotropic light scattering occurs $\left(I_{L}=\right.$ const.), $E$ is simply given by

$$
\mathrm{E}=\frac{4 \pi^{3}}{\lambda^{4}} \eta^{2} \mathrm{~V}_{\mathrm{c}, \mathrm{L}}(0)
$$

$\mathrm{V}_{c}(0)$ is known as the coherence or correlation volume. In case of a system of independently scattering particles, $\mathrm{V}_{\mathrm{c}}(0)$ is identical with the particle volume; for denscly packed systems an interpretation is difficult. Using the invariant

$$
Q=\int_{0}^{\infty} q^{2} \frac{d \Sigma x}{d \Omega}(q) d \Omega=2 \pi^{2} r_{e}^{2} \eta^{2} x
$$

together with eq.2, the X-ray scattering intensity can also be reduced to a q-dependent correlation volume

$$
\mathrm{V}_{\mathrm{c}, \mathrm{X}}(\mathrm{q})=\frac{2 \pi^{2}}{\mathrm{Q}} \frac{\mathrm{d} \Sigma_{\mathrm{X}}}{\mathrm{d} \mathbf{0}}(\mathrm{q}) \text {, }
$$

even if $I_{X}(q)$ is measured in arbitrary units. According to the hierarchical build-up of the aerogel network, $V_{c}(q)$ can be written as

$$
V_{c}(q)=V_{o} \Phi(q) S(q) P(q),
$$

if monodisperse building blocks are assumed with $V_{0}$ being the volume of a primary particle. Due to polydispersity, no oscillations of the scattered intensity are observed in the limiting Porod-regime and a Debye-function is suitable to approximate the particle form factor

$$
P(q, R)=\left(1+\frac{\sqrt{2}}{3} q^{2} R^{2}\right)^{-2}
$$

The constant $\sqrt{2} / 3$ was chosen to let $P(q)$ correspond to the form factor of a perfect sphere at large scattering vectors $\left(P \propto 4.5(q R)^{-4}\right) ; R$ can then be regarded as a sphere radius. During the sol-gel process the primary particles aggregate forming branched clusters, which generally are character- ised by a quantity $D$, the fractal dimension [8]. $D$ describes how the cluster mass scales with the cluster size $\left(M \propto \xi^{D}\right)$ and depends on the process for cluster growth [9]. The fractal behaviour is limited to the range between cluster size $\xi$ and particle radius $R$, which measures up to one order of magnitude on the length scale for medium-density aerogels $\left(100-200 \mathrm{~kg} / \mathrm{m}^{3}\right)$. Thus, the fractal concept should not be overemphasised for such aerogels. Nevertheless, the scaling of the cluster mass shows up in the structure factor $S(q)$, which can be calculated starting off with a correlation function consisting of a power-law term and an exponential cutoff length to account for a limited maximum cluster size $\left(\gamma(r) \propto \mathrm{I}^{\mathrm{D}-3} \exp (-\mathrm{r} / \xi)\right)[10]$ :

$$
\begin{aligned}
S(q, R, \xi, D)=1 & +\frac{D \Gamma(D-1)}{(q R)^{D}}\left(1+\frac{1}{(q \xi)^{2}}\right)^{\frac{1-D}{2}} \\
& \times \sin [(D-1) \arctan (q \xi)],
\end{aligned}
$$

$\Gamma$ denotes the gamma-function. While $S(q)$ shows a broad crossover from the 'fractal' regime $\left(S \propto q^{-D}\right)$ to the constant value $\left(S=\Gamma(D+1)(\xi / R)^{D}\right)$ at smallest scattering vectors, for most of the base-catalysed aerogels a sharp crossover and even a decrease in scattered intensity towards scattering angle zcro is obscrved. This bchaviour can be ascribed to concentration cffects, i.c. closc packing of ncarly monodispersc clusters; the maximum might then be interpretcd as a Bragg-peak of the spacing between neighbouring clusters. A first approximation is to treat the clusters as hard spheres which lcads to a concentration factor of

$$
\Phi(q, L, p)=\frac{1}{1+8 p \Theta(q L)},
$$

with $\Theta(x)$ being the scattering amplitude of a perfect sphere with radius $L$ [11]. In case of monodispersity, $p$ is the packing ratio, i.e. particle volume over the average volume available per particle. Although the clusters have an open structurc, such that thcy can penctrate cach othcr, $\Phi(q)$ is sufficient to empirically fit the data. The single structure functions are depicted schematically in fig. 1 together with

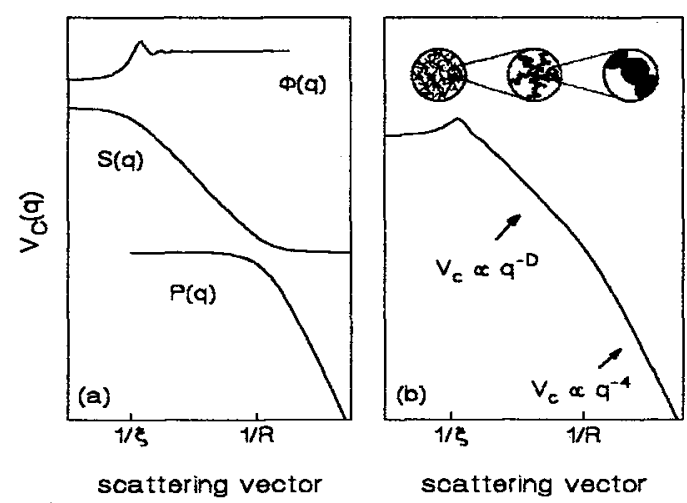

Fig.1: (a) Schematics of the particle form factor $P(q)$, the structure factor $S(q)$ and the concentration factor $\Phi(q)$. (b) The scattered intensity $\mathrm{I}(q) \propto \Phi(q) S(q) P(q)$, which is typical for an aerogel [11]. The different structural features of the gel network (continuum - cluster - particle) show up at the corresponding scattering vectors [1]. 
the resulting scattering function $\mathrm{V}_{\mathrm{c}}(\mathrm{q})$. Additionally, schematics of the different structural features of the gel network are inserted.

The correlation volume, which should be proportional to the optical extinction if both, light and $\mathrm{X}$-rays are scattered by the same inhomogeneities, is thus given by

$$
V_{c}(0)=\frac{4 \pi}{3} \frac{\Gamma(D+1)}{1+8 p} \xi^{D} R^{3-D}
$$

\section{Experimental}

In this study two basically different sample series have been produced: One- and two-step reacted aerogels. In the first, conventional method, wet silica gels were prepared by hydrolysis and condensation of tetramethoxysilane (TMOS) with a stoichiometric amount of water [12]. The target density of the gel was adjusted by an additional amount of methanoI; ammonia was used as catalysts. The catalyst concentration is written as a pseudo $\mathrm{pH}$-value, i.e. $\mathrm{pH}=14$ $\log \left[\mathrm{NH}_{4} \mathrm{OH}\right.$. Two-step gels were prepared by condensation of an acid-catalysed, precondensed silica precursor in acetonitrile, which is induced by addition of the residual amount of water and ammonia [13]. The sample designation reads as follows: $\mathrm{X}-\mathrm{Y} / \mathrm{Z}$, where $\mathrm{X}$ stands for $\mathrm{OS}$ (one-step) or TS (two-step), $\mathrm{Y}$ gives the $\mathrm{pH}-\mathrm{Value}$ and $\mathrm{Z}$ the macroscopic target density in $\mathrm{kg} / \mathrm{m}^{3}$. After about one week of ageing, all gels were supercritically dried with respect to methanol (OS) or with respect to acetonitrile (TS).

A polar nephelometer was employed to probe the angular distribution of the scattered light intensity. A chopped and polarised He-Ne-laser beam is used as a light source $(\lambda=633 \mathrm{~nm})$, the scattered intensity is detected with a photomultiplier mounted on a goniometer For a detailed description of corrections due to the experimental arrangement and the sample geometry see ref.7; the experimental setup for separating surface and bulk scattering contributions is given in ref.4. A commercial double beam spectrometer (PERKIN-ELMER Lambda 9) was employed to determine the optical extinction via transmission measurements in the wavelength range from 300 to $800 \mathrm{~nm}$.

SAXS-measurements were performed at the JUSIFA beam line [14] of the storage ring DORIS III at HASYLAB/DESY (Hamburg) using point collimation $(0.5 \mathrm{x}$ $0.5 \mathrm{~mm}^{2}$ ), an X-ray wavelength of $1.5 \AA$ and two different sample-detector distances $(935 \mathrm{~mm}, 3635 \mathrm{~mm}$ ); thus a $\mathrm{q}$ range from 0.008 to $0.6 \AA^{-1}$ was covered. The high intensity of the incident synchrotron radiation as well as the use of a two-dimensional position-sensitive detector $\left(0.8 \times 0.8 \mathrm{~mm}^{2}\right.$ pixel size) resulted in excellent signal-to-noise ratios within short measurement times.

\section{Results}

Fig.2 depicts the reduced X-ray (SAXS) and light scattering (LS) intensities of three one-step reacted aerogel specimens catalysed at $\mathrm{pH} 9,11$ and 13 . A satisfactory agreement between the level of light scattering by the aerogel bulk and the extrapolated zero-angle SAXS intensity can be observed; thus it is evident that there are no structural inhomogeneities in the bulk beyond the resolution of the small angle $X$-ray scattering experiment. The increase of $V_{C, L}(q)$ at small scattering vectors results from scattering of the external aerogel surface. The accuracy of the normalisation of LS amounts to about $30 \%: 5-10 \%$ experimental error results from the extinction measurement and about $20 \%$ from the determination of $\mathrm{E}^{*}$; the latter is duc to the incompletc mcasurable angular range lcading to errors upon extrapolation (' thin platelet'-geometry, gap between $\theta \approx 75^{\circ}$ and $105^{\circ}$ [41). The error in the SAXS-data due to calibration via the invariant $Q$ is about $4 \%$; thanks to the $q^{2}$-weighting, errors due to extrapolation can be neglected. The LS and SAXS curves of a series of two-step catalysed aerogels with different densities are shown in fig.3: likewise a sufficient agreement is evident. Here, LSdata have been obtained using the 'tile'-geometry which restricts the scattering angle to the range between $30^{\circ}$ and $150^{\circ}[4]$.

All samples show a limiting $\mathrm{q}^{-4}$-behaviour, which is indicative for smooth inner surfaces. The parameters obtained from applying eq. 9 to the SAXS-data are presented in tab.1. The accuracy of packing ratios $p$ is not very high because of the limited $\mathrm{q}_{\min }$ of the SAXS-data. The two-step aerogels were obtained at fixed $\mathrm{pH}$-value, which leads to constant particle radii $\mathbf{R}$ and increasing cluster sizes $\xi$ with decreasing density $\rho$. For the one-step aerogels at constant macroscopic density, both quantities decrease with rising $\mathrm{pH}$-value. The average fluctuation square of the dielectric constant was calculated according to eq. $3 ; \mathrm{E}_{\mathrm{B}}$ is the bulk extinction determined from the angular distribution $I_{L}(q)$ by properly separating the surface scattering contribution $I_{S}(q)$ from the bulk scattering $I_{B}$ which is assumed to be constant.

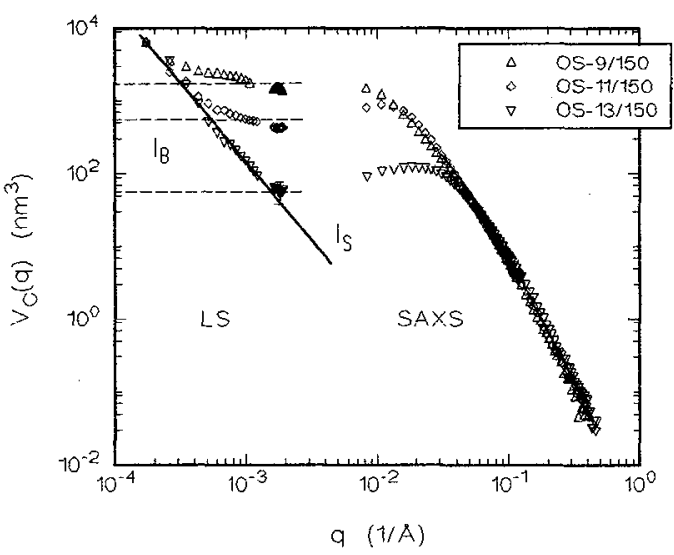

Fig.2: Reduced light (LS) and small angle X-ray (SAXS) scattering intensities $V_{c}(q)$ of three one-step reacted aerogels with same target density $\left(\rho=100 \mathrm{~kg} / \mathrm{m}^{3}\right)$ but different catalyst concentration. Regardless of the gap ranging from $q=0.002 \AA^{-1}$ to $0.08 \Lambda^{-1}$, a satisfactory agreement between light scattering intensity of the aerogel bulk and the extrapolated X-ray intensity is observed. The error bar of about $30 \%$ reflect the uncertainty due to the normalisation procedure for LS; the statistical errors of the SAXS-data are less then one percent. The intensity increase at smallest scattering vectors $q$ is due to light scattering $I_{S}(q)$ from the external aerogel surface (full line), the bulk scattering intensity $I_{R}$ can be regarded as nearly isotropic (dashed lines) [4]. 


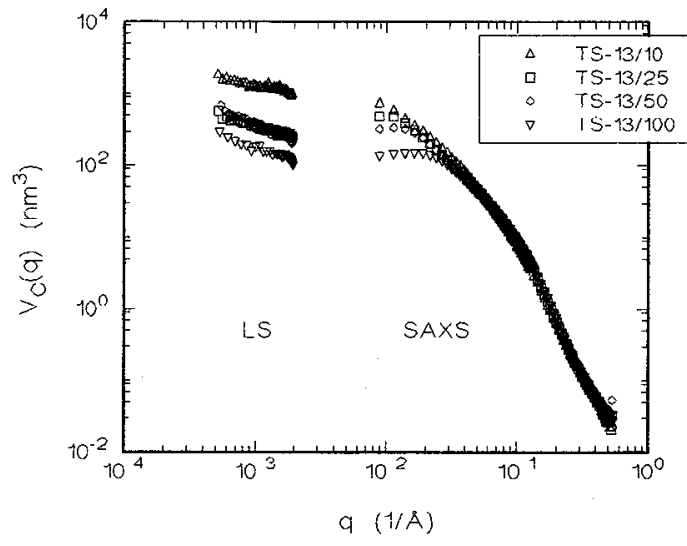

Fig.3: Reduced light (LS) and small angle X-ray (SAXS) scattering intensities $V_{\mathcal{C}}(q)$ of a series of four two-step reacted aerogel with same catalyst concentration but different target densities. All four samples are build-up of primary particles having the same size (crossover at $q \approx 0.1 \AA^{-1}$ ).

\section{Discussion}

The correspondence between reduced light and small angle X-ray scattering data shows that both types of scattering take place at the same inhomogeneities of the aerogel network, described by the normalised correlation function $\gamma(r)$. This allows to directly relate the optical extinction to the quantities of the nanostructure as determined by SAXS and thus to conclude, which structural parameters should be altered to effectively improve the optical transparency: Since the fractal dimension D is an intrinsic property of the cluster growth process and remains nearly constant $(D \approx 2)$ independent of preparation conditions, only particle and cluster sizes can be influenced by varying density and catalyst concentration. The density variation at constant $\mathrm{pH}$-valuc leaves the primary particle size unchanged and leads to $\mathrm{V}_{\mathrm{c}} \propto \xi^{\mathrm{D}} \propto \rho^{\mathrm{D} /(\mathrm{D}-3)}$, where the average density of a single cluster have been used to estimate the macroscopic density $\rho \approx \mathrm{M} / \propto \xi^{\mathrm{D} / \xi^{3}}=\xi^{\mathrm{D}-3}$. For $\mathrm{D}=2, \mathrm{~V}_{\mathrm{C}} \propto \rho^{-2}$ should be expected, however, a much weaker dependence on density is observed for the two-step aerogels investigated. At higher macroscopic densities, concentration effects obviously become more and more

Tab.1: Macroscopic density $\rho\left(\mathrm{kg} \mathrm{m}^{-3}\right)$, the mean fluctuation square $\eta^{2} \mathrm{~L}$, the optical bulk extinction $E_{B}\left(m^{-1}\right)$, LS- and SAXS-derived correlation volume $V_{c, L}(0)$ and $V_{c, X}(0)\left(\mathrm{nm}^{3}\right)$, respectively, particle radius $R(\mathrm{~nm})$, cluster size $\xi(\mathrm{nm})$, fractal dimension $D$ and packing ratio $p$ for the samples investigated.

\begin{tabular}{|c|c|c|r|r|r|c|c|c|c|}
\hline Sample & $\rho$ & $\eta_{L}^{2}$ & $\mathrm{E}_{\mathbf{B}}$ & $\mathrm{V}_{\mathrm{C}, \mathrm{L}}$ & $\mathrm{V}_{\mathbf{c}, \mathrm{X}}$ & $\mathrm{R}$ & $\xi$ & $\mathrm{D}$ & $\mathrm{p}$ \\
\hline OS-9/150 & 220 & 0.117 & 155 & 1719 & 1399 & 3.20 & 15.7 & 2.02 & 0.18 \\
\hline OS-11/150 & 230 & 0.122 & 63.9 & 682 & 840 & 2.13 & 15.0 & 2.24 & 0.35 \\
\hline OS-13/150 & 234 & 0.123 & 6.97 & 73 & 100 & 0.96 & 8.4 & 2.22 & 0.45 \\
\hline TS-13/10 & 13 & 0.007 & 3.73 & 675 & 1013 & 1.25 & 21.1 & 2.13 & 0.29 \\
\hline TS-13/25 & 29 & 0.017 & 1.83 & 141 & 496 & 1.19 & 16.1 & 2.11 & 0.31 \\
\hline TS-13/50 & 67 & 0.038 & 3.52 & 120 & 263 & 1.05 & 13.4 & 2.12 & 0.39 \\
\hline TS-13/100 & 115 & 0.064 & 2.76 & 56 & 99 & 1.07 & 11.2 & 2.08 & 0.73 \\
\hline
\end{tabular}

important. On the other hand, at a given density higher $\mathrm{pH}-$ values diminish both, particle and cluster sizes. A possible explanation for this $\mathrm{pH}$-dependence might be the strongly increased condensation rate: A lot of particles are instantaneously formed at the beginning of the sol-gel process, which must therefore be especially small since the total mass is constant. The same argument holds for the cluster aggregation. Furthermore, reorganisation of mass due to dissolution and reprecipitation phenomena (Ostwald ripening [15]) is negligible because of the drastically shortened gelation time at high pH-values. If in addition the rapid growth results in a narrow cluster size distribution, a higher cluster ordering is favoured resulting in a smaller $\Phi$ (0).

Despite the excellent agreement between LS- and SAXS-data one should bear in mind, that the reduction of $I_{L}(q)$ has been accomplished by calculation of the mean square fluctuation $\eta_{L}^{2}$, assuming the sample to consist of pure silica. Due to the high specific surface area of aerogels, a significant amount of mass with different dielectric properties is present in form of $\mathrm{OH}$-groups and organic residues on the internal surface. If, on the other hand, the SAXS-derived correlation volume $V_{c, X}(q)$ could be precisely determined, $\eta_{L}^{2}$ is calculable from the optical extinction $\mathrm{E}_{\mathrm{B}}$ and information might be obtained on the amount of surface groups.

For the future, SAXS measurements at an ultra small angle scattering beam line are planned, in order to probe smaller scattering vectors and to obtain an overlap between LS and SAXS.

The authors are grateful to the German BMFT/Bonn (contract number 03M2717) for financial support.

\section{References}

[1] J.FRICKE and A.EMMERLING, ' Aerogels - Preparation, Properties and Applications' eds. R.Reisfeld, C.K.Jorgensen, Springer Series Structure and Bonding, Vol.77, Springer Verlag, Berlin (1992), p.37-87.

[2] A.GOETZBERGER and V.WITTWER, Springer Proc. in Phys, 6 (1986) 84.

[3] NOVAKOV, Oral Report, 2nd International Workshop on Transparent Insulation, Freiburg, 1988.

[4] P.WANG, W.KÖRNER, A.EMMERLING, A.BECK, J.KUHN and J.FRICKE, J.Non-Cryst.Solids 145 (1992) 141

[5] P.LINDNER and T.ZEMB, eds., ' Neutron-X-ray and Light Scattering', North-Holland, Amsterdam (1991).

[6] M.KERKER, ' The Scattering of Light', Academic Press, tondon (1969).

[7] A.BECK, R.CAPS and J.FRICKE, J.Phys. D22 (1989) 730 .

[8] B.B.MANDELBROT, 'The Fractal Geometry of Nature', Freeman, San Francisco (1982).

[9] D.W.SCHAEFER, Rev.Phys.Appl. 24-C4 (1988) 121

[10] J.TEDXEIRA, J.Appl.Cryst. 21 (1988) 781.

[11] D.POSSELT, J.S.PEDERSEN and K.MORTENSEN, J.Non-Cryst. Solids 145 (1992) 128.

[12] C.J.BRINKER and G.W.SCHERER, ' Sol-Gel Science', Academic Press, Boston (1990).

[13] T.M.TLLOTSON and L.W.HRUBESH, J.NonCryst. Solids 145 (1992) 44

[14] H.-G.HAUBOLD et al., Rev.Sci.Instrum. 60 (1989) 1943.

[15] R.K.ILER, 'The Chemistry of Silica', John Wiley, New York (1979). 